\title{
Retraction Note: Upregulating microRNA-498 inhibits gastric cancer proliferation invasion and chemoresistance through inverse interaction of Bmi1
}

\author{
Tiancheng Zhao - Yahong Chen · Shihou Sheng $\cdot$ Yuanyu Wu $\cdot$ Tao Zhang
}

Published online: 1 July 2021

(c) The Author(s), under exclusive licence to Springer Nature America, Inc. 2021

Retraction to: Cancer Gene Therapy

https://doi.org/10.1038/s41417-018-0065-7

Published online 25 December 2018

The Editor-in-Chief has retracted this article. Concerns were raised regarding a number of figures, specifically:

Figure 3B: the HGC-27 L-miR498 panel appears to have a number of repeated features

Figure 3B: the AGC L-miR498 panel appears to have a number of repeated features
Figure 5D: the HGC-27 L-miR498 \& pc/+ panel appears to have a number of repeated features

Figure 5E: the AGC L-miR498 \& pc/+ panel appears to have a number of repeated features

The Editor-in-Chief therefore no longer has confidence in the reliability of the data reported in the article. The authors have not responded to correspondence regarding this retraction. 